\title{
Parotid sialometry: A standard technique in the diagnosis of parotid sialadenosis
}

\author{
Magdy A. Riad ${ }^{a}$, Hussain T. Salma ${ }^{\text {, Reda M. I. Sabra }}{ }^{a}$, Tarek A. Hamdya
}

aENT Department, Ain Shams University,

Cairo, Egypt ${ }^{b}$ ENT Department, Dubai Hospital, Dubai, UAE

Correspondence to Magdy A. Riad, 60 Khalifa Mamoun Street, Roxy, Cairo, Egypt e-mail: magdyriad@doctors.org.uk

Received 2 October 2013

Accepted 18 January 2014

The Egyptian Journal of Otolaryngology 2014, 30:138-141

\begin{abstract}
Background
Sialadenosis is a clinical condition characterized by bilateral, painless, diffuse and recurrent parotid gland swelling. Histologically, the only feature of note is acinar cell hypertrophy, with no apparent change in the duct structure or cellular infiltrate. The functional changes in the parotid parenchyma in this condition are ill understood. The association between malnutrition and parotid gland swelling was first reported in Egypt. The pathogenesis of the condition remains unclear.The aim of this study is to explain the development of parotomegaly by analysing the parotid secretory pattern in parotomegaly patients

Subjects and Methods

Patients with malnutrition were studied at Ain Shams University Hospitals. 47 patients, 19 male patients and 28 female patients, were studied, of these eight patients (three male patients and five female patients) suffered parotomegaly. Thirty-one patients with morbid obesity were recruited. 23 patients were female and eight were male. Six patients in this group had parotomegaly. Results were compared with those of 30 healthy volunteers recruited from medical and nursing staff, medical students and patients attending the ENT clinic for minor procedures.Parotid saliva was collected by the standard technique

Results

The results of salivary analysis in 47 patients suggested a pattern of inhibitory proteodyschylia, with increased salivary flow rate and reduced total protein level and amylase activity compared with 30 healthy volunteers. This inhibitory proteodyschylia pattern is identical to the secretory pattern induced experimentally by blocking B-adrenergic receptors in the parotid

Conclusion

A possible explanation for the development of parotomegaly in malnutrition is a degenerative neuropathy affecting the B-adrenergic innervation of the parotid that cause acinar cell congestion by zymogen granules, which is manifested clinically as parotomegaly.
\end{abstract}

\section{Keywords:}

parotomegaly, saliva, sialadenosis

Egypt J Otolaryngol 30:138-141

(c) 2014 The Egyptian Oto - Rhino - Laryngological Society

1012-5574

\section{Introduction}

The association between malnutrition and parotid gland swelling was reported by Kenaway [1] who noted the prevalence of parotomegaly in chronically malnourished patients in Egypt. Similar conditions were described in South and West Africans and in prisoners of war [2].

Clinically, the parotid swelling is of the sialadenotic type - parotomegaly in this condition is bilateral, painless, diffuse and recurrent. In few histologic reports of such parotid swellings, the only feature of note was acinar cell hypertrophy, with no apparent change in the duct structure or cellular infiltrate [3]. The functional changes in the parotid parenchyma in this condition are ill understood.

Biochemical investigation of parotid saliva proved to be useful in the differential diagnosis of parotid swellings [4]. Barton et al. [5] developed a technique for standardizing salivary investigation.
Salivary secretion is controlled by reflex stimulation of the glands with effector autonomic nerve supply. Interruption of the gland innervation at different levels results in consistent changes in the gland parenchyma and salivary secretory pattern and composition [6].

The possibility of salivary parenchymal changes in response to the diet is emphasized by several reports on parotid enlargement resulting from excessive ingestion of starch, and by the fact that individuals predominantly fed on carbohydrate diet have increased salivary amylase activity irrespective of race, and that amylase activity varied within 2 months of dietary modification [7].

It was showed that an increased functional activity of the parotid glands of rats resulted from feeding rats a bulk diet, which caused compensatory enlargement of the parotid gland by an increase in acinar cell size [8]. The changes in the salivary secretory rate and composition in response to the diet have been recognized since Pavlov. 
Salivary amylase is present in low concentration in the saliva of herbivorous animals, but it is almost absent in the saliva of carnivorous animals [9].

The aim of this study was to investigate the aetiology of parotomegaly in malnutrition by examining the parotid salivary secretory pattern in patients with malnutrition and to compare this with the secretory patterns in other groups of nutritional disorders.

\section{Patients and methods}

Patients with malnutrition were studied at Ain Shams University Hospitals. The criteria for inclusion in the study were a body weight of less than $80 \%$ of the ideal weight, a triceps skin fold thickness less than 60\% of the standard and serum albumin level less than $2.9 \mathrm{~g} / 1$ [6]. The mean age of this group was 24.1 years $(\mathrm{SD}=4.6$ years). In all, 47 patients, 19 male patients and 28 female patients, were studied, of these eight patients (three male patients and five female patients) suffered parotomegaly.

Thirty-one patients with morbid obesity were recruited. The mean body weight was $101 \mathrm{~kg}(\mathrm{SD}=18.1 \mathrm{~kg})$, which was more than $30 \%$ of the ideal body weight. The mean age was 27.7 years ( $\mathrm{SD}=6.5$ years), 23 patients were female and eight were male. Six patients in this group had parotomegaly.

Results were compared with those of 30 healthy volunteers recruited from medical and nursing staff, medical students and patients attending the ENT clinic for minor procedures. The mean age of this group was 26.8 years $(\mathrm{SD}=6.3$ years) and the mean weight was $74.3 \mathrm{~kg}(\mathrm{SD}=8.9 \mathrm{~kg})$.

Parotid saliva was collected by the standard technique described by Barton et al. [5] using CarlsonCrittenden cups (Fig. 1). Samples were collected at around 2.00 p.m. to minimize diurnal changes in salivary composition, following refraining from eating and drinking for a period of $2 \mathrm{~h}$. Two separate samples were collected from each individual; saliva was first collected in the resting state for $10 \mathrm{~min}$. The glands were then stimulated by applying $2 \mathrm{ml}$ of $5 \%$ citric acid solution to the dorsum of the tongue, and the stimulated secretion was collected for a further $2 \mathrm{~min}$.

The salivary flow rate was recorded and the samples were then frozen at $-70^{\circ} \mathrm{C}$ until assayed. The salivary amylase activity was determined by Deutsches Arzneibuch assay and the total salivary protein was determined by the pierce technique. Salivary electrolytes levels were measured by flame photometry and immunoglobulins were estimated by an enzyme-linked immunosorbent assay technique using affinity-purified antihuman immunoglobulins (Tables 1 and 2).

\section{Results}

The results were independent of age or sex. No significant interindividual variations were noted.

\section{The malnutrition group}

The parotid salivary flow rate was increased in the malnutrition group, both in the resting and stimulated states. The stimulated flow rate of the parotomegaly sialadenotic group was also significantly higher than the control level, only in the stimulated state, which can help in establishing diagnosis, and suggests that certain functional changes develop in the parotid with malnutrition.

\section{Figure 1}

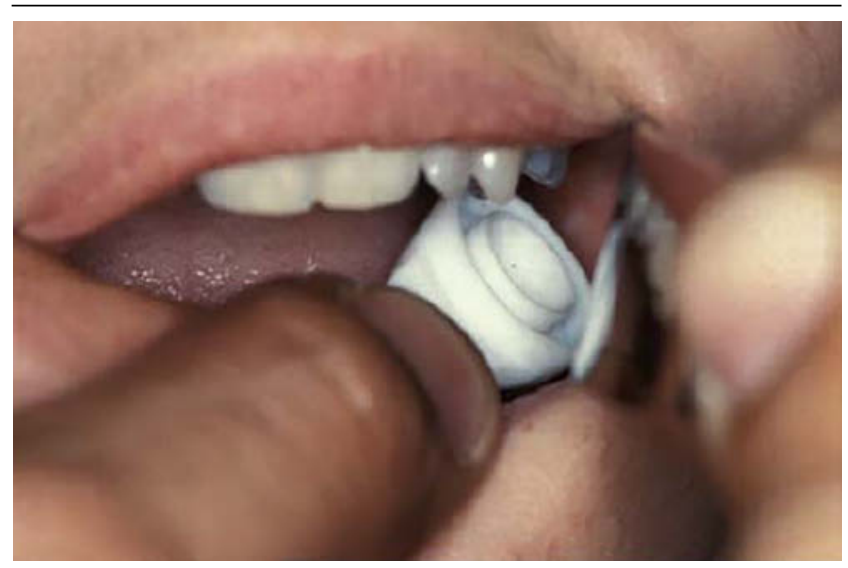

Carlson-Crittenden parotid cups.

Table 1 Resting and stimulated parotid salivary flow rate

\begin{tabular}{lccc}
\hline Group & $n$ & Resting flow rate $(\mathrm{ml} / \mathrm{min})$ & Stimulated flow rate $(\mathrm{ml} / \mathrm{min})$ \\
\hline Control & 30 & $0.07(\mathrm{SD}=0.02)$ & $0.65(\mathrm{SD}=0.2)$ \\
Malnutrition & 39 & $0.085(\mathrm{SD}=0.025)^{\star}$ & $0.92(\mathrm{SD}=0.42)^{\star \star}$ \\
Sialadenosis in malnutrition & 8 & $0.075(\mathrm{SD}=0.025)$ & $0.9(\mathrm{SD}=0.46)^{\star}$ \\
Morbid obesity & 25 & $0.08(\mathrm{SD}=0.02)$ & $0.86(\mathrm{SD}=0.37)^{\star \star}$ \\
Sialadenosis in obesity & 6 & $0.065(\mathrm{SD}=0.03)$ & $0.96(\mathrm{SD}=0.17)^{\star}$ \\
\hline
\end{tabular}

${ }^{*} P<0.05,{ }^{* *} P<0.01$. 
Table 2 Resting and stimulated parotid salivary total protein and amylase

\begin{tabular}{lccccc}
\hline Group & $n$ & $\begin{array}{c}\text { Resting salivary total } \\
\text { protein }(\mathrm{mg} / \mathrm{dl})\end{array}$ & $\begin{array}{c}\text { Stimulated salivary } \\
\text { total protein }(\mathrm{mg} / \mathrm{dl})\end{array}$ & $\begin{array}{c}\text { Resting salivary } \\
\text { amylase activity }(\mathrm{AU} / \mathrm{dl})\end{array}$ & $\begin{array}{c}\text { Stimulated salivary } \\
\text { amylase activity }(\mathrm{AU} / \mathrm{dl})\end{array}$ \\
\hline Control & 30 & $203(\mathrm{SD}=36)$ & $157(\mathrm{SD}=29)$ & $19.7(\mathrm{SD}=2.6)$ & $21.6(\mathrm{SD}=2.9)$ \\
Malnutrition & 39 & $185(\mathrm{SD}=29)^{*}$ & $139(\mathrm{SD}=21)^{\star *}$ & $18.9(\mathrm{SD}=2.9)$ & $21.1(\mathrm{SD}=2.8)$ \\
Sialadenosis in malnutrition & 8 & $189(\mathrm{SD}=21)$ & $132(\mathrm{SD}=21)^{\star}$ & $19.3(\mathrm{SD}=2.2)$ & $18.3(\mathrm{SD}=2.9)^{\star *}$ \\
Morbid obesity & 25 & $206(\mathrm{SD}=41)$ & $154(\mathrm{SD}=28)$ & $21.4(\mathrm{SD}=3.3)^{\star}$ & $23.9(\mathrm{SD}=3.2)^{\star}$ \\
Sialadenosis in obesity & 6 & $209(\mathrm{SD}=35)$ & $156(\mathrm{SD}=26)$ & $22.1(\mathrm{SD}=2.8)^{*}$ & $24.2(\mathrm{SD}=3.1)^{\star}$ \\
\hline
\end{tabular}

${ }^{\star} P<0.05,{ }^{* *} P<0.01$.

Total salivary protein was reduced in the malnutrition group, both in the resting and stimulated states. Only the stimulated salivary protein level was reduced significantly in the sialadenotic group.

The amylase activity of stimulated secretions was significantly reduced in the malnourished patients who developed parotomegaly. The rest of the malnourished patients who have not developed parotid swelling have an amylase activity within normal limits. Reduction in amylase secretion in parotomegaly indicates acinar cell pathology; it is possible that parotomegaly develops because of increase in acinar cells diameter, caused by arrest of zymogen granules secretion.

\section{The obesity group}

In contrast, the salivary amylase activity of obese patients was significantly increased, both in the resting and stimulated saliva, indicating hyperactivity of the glandular secretory system or a neural facilitation. The flow rate in the stimulated state was increased in the obese patients. No changes were noted in the salivary protein in the obese group.

No significant difference in the immunoglobulins or electrolytes levels was observed between patients and controls. This excludes the possibility of chronic inflammation as the cause of parotomegaly. The normal electrolyte level indicates that the tubular cells functions are normal, as most of the electrolytes secretion and absorption processes are conducted in the tubules, and are not controlled by the autonomic nerves. The fact that the changes in the salivary pattern are mainly in the amylase and protein secretion suggests that the primary abnormality in the parotid of patients with nutritional disorders affects the acinar cells and its neurogenic control.

\section{Discussion}

Sialadenosis is a noninflammatory disease of the salivary glands. It is observed in association with endocrine disorders, malnutrition and neurological diseases. The parotid glands are predominantly affected by a parenchymatous swelling. On the basis of the morphologic and biochemical investigations, it can be assumed that disorders of the glands acinar protein secretion are responsible for sialadenosis. The changes in secretory behaviour are caused by either excessive stimulation (stimulatory proteodyschylia) or inhibition of secretion (inhibitory proteodyschylia). The origin of these secretory disorders is very likely to be found in the autonomic nerve system. The pathophysiology of sialadenosis can therefore only be understood after elucidation of the physiological mechanisms of salivary gland secretion [10].

The results of this study show that parotid salivation is disturbed in malnutrition and in obesity; the shift from normality is exaggerated when the glands swell up causing parotomegaly. The importance of such a significant shift from normal secretory pattern is that it helps in explaining the pathogenesis of the disorder.

The parotid secretory pattern in malnourished patients is characterized by increased salivary flow rate and reduced total protein. The reduction in salivary amylase activity was significant only in the stimulated state of the parotomegaly group. In the morbidly obese group, the parotid salivary amylase activity was significantly increased, and the salivary flow rate was only increased after stimulation.

The reduction in the total protein secreted in malnourished patients may be related to the observed increase in the flow rate, the associated low serum protein or a disorder of the protein secretory mechanism in the glands. The finding of normal or reduced amylase activity suggests that the gland swelling in malnourished patients was not induced by the relative carbohydrate predominance in the diet, where an increase in the amylase activity would be expected [3].

The immunoglobulin levels were within normal limits, which is in accordance with several reports. This excludes the possible role of associated chronic infections in the development of parotomegaly in malnourished patients [11].

Parotid gland enlargement occurs in about $25 \%$ of patients with bulimia nervosa. Bulimia patients had a reduced resting parotid salivary flow rate. Bulimia 
patients who developed sialadenosis had reduced resting and stimulated flow rates. The salivary amylase activity was increased in both the resting and stimulated states in bulimia patients and in the sialadenosis group. The resting total protein levels were greater in bulimia patients. The electrolyte and immunoglobulin levels were within normal limits [4].

The possibility of protein and enzymatic secretory disturbances due to autonomic nerve disorders as an explanation for the development of sialadenosis in bulimia nervosa was postulated. Few years later, a cholinomimetic medication was tried in bulimia patients, and resolution of the parotid gland enlargement in bulimia patients was confirmed. Pilocarpine hydrochloride drops were administered orally at a dosage of $1.25-5.0 \mathrm{mg} /$ day until the parotid gland enlargement was significantly reduced [12].

The result of this study is in accordance with the suggestion that the development of parotomegaly in obese patients is because of work hypertrophy and is related to diet. The significant changes in the salivary flow rate and amylase activity also indicate that parotomegaly in obese patients is not simply related to glandular fat infiltration, but that some changes in the functional activity of the glands had developed.

The possible explanation for the development of parotomegaly in malnourished patients is a degenerative neuropathy of the autonomic nerve supply of the gland caused by poor diet and avitaminosis. Autonomic neuropathy is known to occur in several metabolic conditions and may be the primary aetiological factor in the development of sialadenosis.

A degenerative $\mathrm{B}$-adrenergic neuropathy, induced experimentally in animal models, produced an inhibitory form of parotid secretion (proteodyschylia) characterized by reduced salivary protein and amylase activity associated with increased flow rate. This form of inhibitory proteodyschylia [8] is identical to the secretory pattern in malnourished patients and may be the cause of acinar cell congestion by zymogen granules, which is manifested clinically as parotomegaly.

\section{Acknowledgements \\ Conflicts of interest}

None declared.

\section{References}

1 Kenaway MR. Endemic enlargement of parotid gland in Egypt. Trans R Soc Trop Med Hyg 1937; 31:339-344.

2 Du Plessis DJ. Parotid enlargement in malnutrition. S Afr Med J 1956; 30:700-704.

3 Sandstead HR, Kohen CJ, Sessians SM. Enlargement of parotid gland in malnutrition. Am J Clin Nutr 1955; 3:198-203.

4 Riad MA, Barton JR, Wilson JW, Maran AGD. Parotid salivary secretory pattern in bulimia nervosa. Acta Otolaryngol 1991; 111:925-928.

5 Barton R, Riad MA, Gaze M, Maran AGD. Mucosal immunodeficiency in smokers and in patients with epithelial head and neck tumours. Gut 1990; 31:181-186.

6 Squires BT. Human salivary amylase secretion in relation to diet. J Physiol 1953; 119:153-157.

7 Reddy N, Raghvramulu N, Bhaskaram M. Secretory IgA in protein calorie malnutrition. Arch Dis Child 1976; 51:82.

8 Arglebe C, Eysholdt V, Chilla R. Pharmacological inhibition of salivary glands. Effect of experimentally induced B-receptor block on the rat parotid gland. ORL J Otorhinolaryngol Relat Spec 1976; 38:218-229.

9 Mignogna MD, Fedele S, Lo Russo L. Anorexia/bulimia-related sialadenosis of palatal minor salivary glands. J Oral Pathol Med 2004; 33:441-442.

10 Chilla R. Sialadenosis of the salivary glands of the head. Studies on the physiology and pathophysiology of parotid secretion. Adv Otorhinolaryngol $1981 ; 26: 1-38$.

11 Satoh M, Yoshihara T. Clinical and ultracytochemical investigation of sialadenosis. Acta Otolaryngol Suppl 2004; 553:122-127.

12 Mehler PS, Wallace JA. Sialadenosis in bulimia: a new treatment. Arch Otolaryngol Head Neck Surg 1993; 119:787-788. 